\title{
On Initial Data Problem for a Periodic Two-Component b-Family System
}

\section{Xiaohuan Wang ${ }^{1}$}

Received: 22 October 2021 / Accepted: 17 January 2022/ Published online: 28 January 2022

(c) The Author(s) 2022, corrected publication 2022

\section{Abstract}

This paper is concerned with the initial date problem for a periodic two-component b-family system. We prove that the solution map of the Cauchy problem of the b-family system is not uniformly continuous in $H^{s}(\mathbb{S}), s>5 / 2$.

Keywords Non-uniform dependence $\cdot b$-Family system $\cdot$ Energy estimates $\cdot$ Initial value problem

Mathematics Subject Classification $35 \mathrm{G} 25 \cdot 35 \mathrm{~B} 30 \cdot 35 \mathrm{~L} 05$

\section{Abbreviations}

$\mathbb{S} \quad(0,1)$;

$f \gtrsim g \quad$ Meas that there exists a positive constant $C$ such that $f \geq C g$ (similarly for $f \lesssim g)$.

\section{Introduction}

In this paper, we study the periodic case of following nonlinear dispersive system [1]

$$
\begin{cases}m_{t}=u m_{x}+k_{1} m u_{x}+k_{2} \rho \rho_{x}, & t>0, x \in \mathbb{R}, \\ \rho_{t}=k_{3}(\rho u)_{x}, & t>0, x \in \mathbb{R}, \\ m(0, x)=m_{0}(x), \rho(0, x)=\rho_{0}(x), & x \in \mathbb{R},\end{cases}
$$

Xiaohuan Wang

003304@ nuist.edu.cn

1 College of Mathematics and Statistics, Nanjing University of Information Science and Technology, Nanjing 210044, People's Republic of China 
where $m=u-u_{x x}$. Lin-Yin [2] obtained the local well-posedness of system (1). Zou [3] considered the system (1) and obtained some properties of the solutions. (1) is also considered by $\mathrm{Zhu}$ and $\mathrm{Xu}$ [4] in Besov space.

If $\rho \equiv 0$, system (1) will become a number of the $b$-family equation

$$
u_{t}-u_{t x x}+c_{0} u_{x}+(b+1) u u_{x}+\sigma u_{x x x}=b u_{x}+u u_{x x x},
$$

where $c_{0}, b$ and $\sigma$ are constants. For any $b \neq-1$, (2) can be derived as the family of asymptotically equivalent shallow water wave equation that emerge at quadratic order accuracy by an appropriate Kodama transformation, see [5, 6] for the details. For $b=-1$, the corresponding Kodama transformation is singular and the asymptotic ordering is violated, see $[5,6]$.

If $b=2$ and $\sigma=0$, (2) will become the Camassa-Holm equation [7]. The authors in [8-11] studied the Cauchy problem of the Camassa-Holm equation. If $b=3$ and $c_{0}=\sigma=0$, similarly, (2) will become the Degasperis-Procesi equation [12], which has been studied by $[2,13]$.

If $\rho \not \equiv 0$ and if $k_{1}=2$, the system (1) will become the two-component Camassa-Holm system $[14,15]$

$$
\begin{cases}m_{t}+u m_{x}+2 m u_{x}+\sigma \rho \rho_{x}=0, & t>0, x \in \mathbb{R}, \\ \rho_{t}+(\rho u)_{x}=0, & t>0, x \in \mathbb{R},\end{cases}
$$

where $m=u-u_{x x}$ and $\sigma= \pm 1$. When $\sigma=1$, local well-posedness of system (3) was established by $[15,16]$.

As for Camassa-Holm equation, it is more important to study the properties of solutions, such as the persistence properties and unique continuation of solutions, non-uniform dependence on initial data, see [17-23] for details. In this paper, we will consider the non-uniform dependence on initial data to periodic system (1), that is

$$
\begin{cases}m_{t}=u m_{x}+k_{1} m u_{x}+k_{2} \rho \rho_{x}, & t>0, x \in \mathbb{R}, \\ \rho_{t}=k_{3}(\rho u)_{x}, & t>0, x \in \mathbb{R}, \\ m(0, x)=m_{0}(x), \rho(0, x)=\rho_{0}(x), & x \in \mathbb{R}, \\ u(t, x+1)=u(t, x), \rho(t, x+1)=\rho(t, x), & t \geq 0, x \in \mathbb{R} .\end{cases}
$$

Denote $\rho=\left(1-\partial_{x}^{2}\right)\left(\bar{\rho}-\bar{\rho}_{0}\right)$, and $\bar{\rho}$ follows from the pointwise density $\rho$ by convolution against the Green's function of the Helmholtz operator $1-\partial_{x}^{2}$ (cf. [14]). If $\gamma=\bar{\rho}-\bar{\rho}_{0}$, then system (1) is called as modified Camassa-Holm system. We remark that there is significant difference between system (1) and (3) with $\rho=\gamma-\gamma_{x x}$. It is easy to see that when $\rho=\gamma-\gamma_{x x}$, there are some similar properties between the two equations in system (3). Thus the proof of non-uniform dependence on initial data to system (3) with $\rho=\gamma-\gamma_{x x}$ is similar to the signal equation, for example, Camassa-Holm equation. But in system (1), $\rho$ and $u$ have different properties, see Theorem 2.1. This needs construct different asymptotic solution, see Sect. 3. On the other hand, in order to obtain the non-uniform dependence on initial data to system (1), 
we must modify the earlier method used in [19, 22]. In papers [19, 22], they estimated the $H^{1}$-norm of the difference between the approximate and actual solutions, but it does not work for system (1). It is easy to see that the method used in [19, 22] is available only when $k_{1}=2$ and $k_{2}=k_{3}$. In this paper, there is not any restriction about the parameters $k_{1}, k_{2}$ and $k_{3}$. Comparing with [24], there are some difference. Firstly, in [24], we considered the whole space, which is different from the periodic case. And so the approximate solutions constructed in this paper is different from [24]. Secondly, we take different norm from [24], see [24, Theorems 3.1 and 4.1]. Lastly, the model considered in [24] is a special case of (1).

This paper is organized as follows. In Sect. 2, we obtain the well-posedness of system (1) by using Kato's theory and then use it to prove the basic energy estimate from which we derive a lower bound for the lifespan of the solution as well as an estimate of the $H^{s}(\mathbb{S}) \times H^{s-1}(\mathbb{S})$ norm of the solution $(u(t, x), \rho(t, x))$ in terms of $H^{s}(\mathbb{S}) \times H^{s-1}(\mathbb{S})$ norm of the initial data $\left(u_{0}, \rho_{0}\right)$, where $\mathbb{S}=(0,1)$. In Sect. 3 , we construct approximate solutions, compute the error and estimate the $H^{\mu}$-norm of this error. In Sect. 4 , we estimate the difference between approximate and actual solutions, where the exact solution is a solution to system (1) with initial data given by the approximate solutions evaluated at time zero. The non-uniform dependence on initial data for system (1) is established in Sect. 5 by construct two sequences of solutions to (1) in a bounded subset of the Sobolev space $H^{s}(\mathbb{S}) \times H^{s-1}(\mathbb{S})$, whose distance at the initial time is converging to zero while at any later time it is bounded below by a positive constant.

\section{Local Well-Posedness}

In this section we apply the Kato's theory [25] to establish the local well-posedness of (1). Set $\Lambda=\left(1-\partial_{x}^{2}\right)^{1 / 2}$ and $G(x)=\frac{\cosh \left(x-[x]-\frac{1}{2}\right)}{2 \sinh \left(\frac{1}{2}\right)}$, where $[x]$ stands for the integer part of $x$. Then the operator $\Lambda^{-2}$ can be expressed as

$$
\Lambda^{-2} f(x)=(G * f)(x)=\frac{1}{2} \int_{0}^{1} \frac{\cosh \left(x-y-[x-y]-\frac{1}{2}\right)}{\sinh \left(\frac{1}{2}\right)} f(y) \mathrm{d} y, \quad f \in L^{2}(\mathbb{S}),
$$

where $G(x)$ is the Green's function associated with $\Lambda^{-2}$. Hence (4) is equivalent to the following system

$$
\begin{cases}u_{t}-u u_{x}=\partial_{x} \Lambda^{-2}\left(\frac{k_{1}}{2} u^{2}+\frac{3-k_{1}}{2} u_{x}^{2}+\frac{k_{2}}{2} \rho^{2}\right), & t>0, x \in \mathbb{R}, \\ \rho_{t}-k_{3} u \rho_{x}=k_{3} u_{x} \rho, & t>0, x \in \mathbb{R}, \\ u(0, x)=u_{0}(x), \rho(0, x)=\rho_{0}(x), & x \in \mathbb{R}, \\ u(t, x+1)=u(t, x), \rho(t, x+1)=\rho(t, x), & t \geq 0, x \in \mathbb{R} .\end{cases}
$$


In the rest of this paper, for convenience, we rewrite the above system as

$$
\begin{cases}u_{t}-u u_{x}=\partial_{x} \Lambda^{-2}\left(\frac{k_{1}}{2} u^{2}+\frac{3-k_{1}}{2} u_{x}^{2}+\frac{k_{2}}{2} \rho^{2}\right), & t>0, x \in \mathbb{S}, \\ \rho_{t}-k_{3} u \rho_{x}=k_{3} u_{x} \rho, & t>0, x \in \mathbb{S}, \\ u(0, x)=u_{0}(x), \rho(0, x)=\rho_{0}(x), & x \in \mathbb{S}\end{cases}
$$

Let

$$
\begin{aligned}
z & :=\left(\begin{array}{c}
u \\
\rho
\end{array}\right), \quad A(z):=\left(\begin{array}{cc}
-u \partial_{x} & 0 \\
0 & -k_{3} u \partial_{x}
\end{array}\right), \\
f(z) & :=\left(\begin{array}{c}
\partial_{x} \Lambda^{-2}\left(\frac{k_{1}}{2} u^{2}+\frac{3-k_{1}}{2} u_{x}^{2}+\frac{k_{2}}{2} \rho^{2}\right) \\
k_{3} u_{x} \rho
\end{array}\right),
\end{aligned}
$$

then the first two equations of system (5) can be replaced by

$$
\frac{d z}{d t}+A(z) z=f(z)
$$

One can mimic the method of [2] to verify that all the assumptions of the Kato's theory holds for system (5). Thus we have the following result.

Theorem 2.1 Given $z_{0}=\left(u_{0}, \rho_{0}\right) \in H^{s} \times H^{s-1}, s \geq 2$. Then there exists a maximal existence time $T=T\left(\left\|z_{0}\right\|_{H^{s} \times H^{s-1}}\right)>0$ and a unique solution $z=(u, \rho)$ to system $(5)$ such that

$$
z=z\left(\cdot, z_{0}\right) \in C\left([0, T) ; H^{s} \times H^{s-1}\right) \cap C^{1}\left([0, T) ; H^{s-1} \times H^{s-2}\right) .
$$

Moreover, the solution depends continuously on the initial data, i.e. the mapping

$$
z_{0} \rightarrow z\left(\cdot, z_{0}\right): H^{s} \times H^{s-1} \rightarrow C\left([0, T) ; H^{s} \times H^{s-1}\right) \cap C^{1}\left([0, T) ; H^{s-1} \times H^{s-2}\right)
$$

is continuous.

Next, we want to get the lower bounded of the maximal existence time $T$. Meanwhile, we will prove that there exists a positive constant $T_{0}$ such that the $H^{s}$-norm of the solution will be dominated by the $H^{s}$-norm of the initial data. The following lemmas are needed.

Lemma 2.1 [26] If $r>0$, then $H^{r} \cap L^{\infty}$ is an algebra. Moreover,

$$
\|f g\|_{H^{r}} \leq C\left(\|f\|_{\infty}\|g\|_{H^{r}}+\|f\|_{H^{r}}\|g\|_{\infty}\right)
$$

where $C$ is a positive constant depending only on $r$.

Lemma 2.2 [26] If $r>0$, then 


$$
\left\|\left[\Lambda^{r}, f\right] g\right\|_{2} \leq C\left(\left\|f_{x}\right\|_{\infty}\left\|\Lambda^{r-1} g\right\|_{2}+\left\|\Lambda^{r} f\right\|_{2}\|g\|_{\infty}\right),
$$

where $C$ is a positive constant depending only on $r$.

Due to the proof of the following is similar to that in [23], we only give the outline of proof.

Theorem 2.2 Let $s>\frac{5}{2}$. If $z=(u, \rho)$ is a solution of system (5) with initial data $z_{0}$ described in Theorem 2.1, then the maximal existence time $T$ satisfies

$$
T \geq T_{0}:=\frac{1}{2 C_{s}\left\|z_{0}\right\|_{H^{s} \times H^{s-1}}},
$$

where $C_{s}$ is a constant depending only on s. Also, we have

$$
\|z(t)\|_{H^{s} \times H^{s-1}} \leq 2\left\|z_{0}\right\|_{H^{s} \times H^{s-1}}, \quad 0 \leq t \leq T_{0} .
$$

Proof Similar to [23], it is not hard to get that

$$
\frac{1}{2} \frac{d}{d t}\|z(t)\|_{H^{s} \times H^{s-1}}^{2} \leq C_{s}\|z(t)\|_{H^{s} \times H^{s-1}}^{3}, \quad 0 \leq t<T .
$$

Integrating (8) from 0 to $t$, we have

$$
\|z(t)\|_{H^{s} \times H^{s-1}} \leq \frac{\left\|z_{0}\right\|_{H^{s} \times H^{s-1}}}{1-C_{s}\left\|z_{0}\right\|_{H^{s} \times H^{s-1}} t} .
$$

The above inequality implies that $\|z(t)\|_{H^{s} \times H^{s-1}}$ is finite if $C_{s}\left\|z_{0}\right\|_{H^{s} \times H^{s-1}} t<1$. Let $T_{0}=\frac{1}{2 C_{s}\left\|z_{0}\right\|_{H^{s} \times H^{s-1}}}$, then we get

$$
\|z(t)\|_{H^{s} \times H^{s-1}} \leq \frac{\left\|z_{0}\right\|_{H^{s} \times H^{s-1}}}{1-C_{s}\left\|z_{0}\right\|_{H^{s} \times H^{s-1}} T_{0}}=2\left\|z_{0}\right\|_{H^{s} \times H^{s-1}}, \quad 0 \leq t \leq T_{0} .
$$

The proof is complete.

Recall that $\|z(t)\|_{H^{s} \times H^{s-1}}^{2}=\|u(t)\|_{H^{s}}^{2}+\|\rho(t)\|_{H^{s-1}}^{2}$, where $z(t)=(u(t), \rho(t))$. It follows from Theorem 2.2 that

$$
\|u(t)\|_{H^{s}},\|\rho(t)\|_{H^{s-1}} \leq\|z(t)\|_{H^{s} \times H^{s-1}} \leq 2\left\|z_{0}\right\|_{H^{s} \times H^{s-1}}, \quad 0 \leq t \leq T_{0} .
$$

\section{Approximate Solutions}

In this section we first construct a two-parameter family of approximate solutions by using a similar method to [20], then compute the error and last estimate the $H^{\mu}$ -norm of the error $\left(1<\mu<\frac{3}{2}\right)$. Throughout this paper, we assume that $s>5 / 2$ and $1<\mu<s-1$. 
Following [20], our approximate solutions $u^{\omega, \lambda}=u^{\omega, \lambda}(t, x)$ and $\rho^{\omega, \lambda}=\rho^{\omega, \lambda}(t, x)$ to (5) will be consist of a low frequency and a high frequency part, i.e.

$$
u^{\omega, \lambda}=\omega \lambda^{-1}+\lambda^{-s} \cos (\lambda x+\omega t), \quad \rho^{\omega, \lambda}=\omega \lambda^{-1}+\lambda^{-s+1} \cos \left(\lambda x+k_{3} \omega t\right),
$$

where $\omega$ is in a bounded set of $\mathbb{S}$ and $\lambda$ is in the set of positive integers $\mathbb{Z}_{+}$.

Direct calculation shows that

$$
\begin{aligned}
u_{t}^{\omega, \lambda}-u^{\omega, \lambda} u_{x}^{\omega, \lambda} & =\frac{1}{2} \lambda^{-2 s+1} \sin (2 \lambda x+2 \omega t) \\
: & =E_{1},
\end{aligned}
$$

and

$$
\begin{aligned}
\partial_{x} \Lambda^{-2} & \left(\frac{k_{1}}{2}\left(u^{\omega, \lambda}\right)^{2}+\frac{3-k_{1}}{2}\left(u_{x}^{\omega, \lambda}\right)^{2}+\frac{k_{2}}{2}\left(\rho^{\omega, \lambda}\right)^{2}\right) \\
= & -\frac{k_{1}}{2} \lambda^{-2 s+1} \Lambda^{-2} \sin (2 \lambda x+\omega t)-k_{1} \omega \lambda^{-s} \Lambda^{-2} \sin (\lambda x+\omega t)+\frac{3-k_{1}}{2} \lambda^{-2 s+3} \Lambda^{-2} \sin (2 \lambda x+2 \omega t) \\
& -k_{2} \omega \lambda^{-s+1} \Lambda^{-2} \sin \left(\lambda x+k_{3} \omega t\right)-\frac{k_{2}}{2} \lambda^{-2 s+3} \Lambda^{-2} \sin \left(2 \lambda x+2 k_{3} \omega t\right) \\
:= & E_{2}+E_{3}+E_{4}+E_{5}+E_{6} .
\end{aligned}
$$

Similarly, we have

$$
\begin{aligned}
& \rho_{t}^{\omega, \lambda}-k_{3} u^{\omega, \lambda} \rho_{x}^{\omega, \lambda}-k_{3} u_{x}^{\omega, \lambda} \rho^{\omega, \lambda} \\
& \left.\quad=k_{3} \lambda^{-s} \sin (\lambda x+\omega t)+k_{3} \lambda^{-2 s+2} \sin \left(2 \lambda x+\left(k_{3}+1\right) \omega t\right)\right) \\
& \quad:=F_{1}+F_{2} .
\end{aligned}
$$

Let $C$ be a generic positive constant. For any positive quantities $P$ and $Q$, we write $P \lesssim Q(P \gtrsim Q)$ means that $P \leq C Q(P \geq C Q)$ in the following.

Next, we estimate the error. We need the following lemma.

Lemma 3.1 [20] Let $\sigma \in \mathbb{R}$. If $\lambda \in \mathbb{Z}_{+}$and $\lambda \gg 1$ then

$$
\|\cos (\lambda x-\alpha)\|_{H^{\sigma}(\mathbb{S})} \approx \lambda^{\sigma}, \quad \alpha \in \mathbb{R} .
$$

The above relation also holds if $\cos (\lambda x-\alpha)$ is replaced by $\sin (\lambda x-\alpha)$.

Estimating the $H^{\mu}$-norm of $E_{1}$. By Lemma 3.1 and $\mu \leq s-1$, we have

$$
\begin{aligned}
\left\|E_{1}\right\|_{H^{\mu}} & =\left\|\frac{1}{2} \lambda^{-2 s+1} \sin (2 \lambda x+2 \omega t)\right\|_{H^{\mu}} \\
& \lesssim \mid \lambda^{-2 s+\mu+1} \lesssim \lambda^{-s} .
\end{aligned}
$$

Estimating the $H^{\mu}$-norms of $E_{2}-E_{6}$. Also, we have 


$$
\begin{aligned}
\left\|E_{2}\right\|_{H^{\mu}} & =\left|\frac{k_{1}}{2}\right| \cdot\left\|\lambda^{-2 s+1} \Lambda^{-2} \sin (2 \lambda x+2 \omega t)\right\|_{H^{\mu}} \\
& \lesssim \lambda^{-2 s+1}\|\sin (2 \lambda x+2 \omega t)\|_{H^{\mu-2}} \lesssim \lambda^{-2 s+\mu-1}, \\
\left\|E_{3}\right\|_{H^{\mu}} & =\left|k_{1}\right| \cdot\left\|2 \omega \lambda^{-s} \Lambda^{-2} \sin (\lambda x+\omega t)\right\|_{H^{\mu}} \\
& \lesssim \lambda^{-s}\|\sin (\lambda x+\omega t)\|_{H^{\mu-2}} \lesssim \lambda^{-s+\mu-2}, \\
\left\|E_{4}\right\|_{H^{\mu}} & =\left|\frac{3-k_{1}}{2}\right| \cdot\left\|\frac{1}{2} \lambda^{-2 s+3} \Lambda^{-2} \sin (2 \lambda x+2 \omega t)\right\|_{H^{\mu}} \\
& \lesssim \lambda^{-2 s+3}\|\sin (2 \lambda x+2 \omega t)\|_{H^{\mu-2}} \lesssim \lambda^{-2 s+\mu+1}, \\
\left\|E_{5}\right\|_{H^{\mu}} & =\left|k_{2}\right| \cdot\left\|\omega \lambda^{-s+1} \Lambda^{-2} \sin \left(\lambda x+k_{3} \omega t\right)\right\|_{H^{\mu}} \\
& \lesssim \lambda^{-s+1}\left\|\sin \left(\lambda x+k_{3} \omega t\right)\right\|_{H^{\mu-2}} \lesssim \lambda^{-s+\mu-1}, \\
\left\|E_{6}\right\|_{H^{\mu}} & =\left|\frac{k_{2}}{2}\right| \cdot\left\|\frac{1}{2} \lambda^{-2 s+3} \Lambda^{-2} \sin \left(2 \lambda x+2 k_{3} \omega t\right)\right\|_{H^{\mu}} \\
& \lesssim \lambda^{-2 s+3}\left\|\sin \left(2 \lambda x+2 k_{3} \omega t\right)\right\|_{H^{\mu-2}} \lesssim \lambda^{-2 s+\mu+1} .
\end{aligned}
$$

Similarly, we can estimate the $H^{\mu-1}$-norm of $F_{1}$ and $F_{2}$

$$
\begin{aligned}
& \left.\| k_{3} \lambda^{-s} \sin (\lambda x+\omega t)+k_{3} \lambda^{-2 s+2} \sin \left(2 \lambda x+\left(k_{3}+1\right) \omega t\right)\right) \|_{H^{\mu-1}} \\
& \quad \lesssim \lambda^{-s+\mu-1}+\lambda^{-2 s+\mu+1} .
\end{aligned}
$$

Collecting all error estimates together, we have the following theorem.

Theorem 3.1 Let $s>\frac{5}{2}$ and $1<\mu<\frac{3}{2}$. When $\omega$ is in a bounded set of $\mathbb{R}$ and $\lambda \gg 1$, we have that

$$
\|E\|_{H^{\mu}},\|F\|_{H^{\mu-1}} \lesssim \lambda^{-r_{s}}, \quad \text { for } \lambda \gg 1,0<t<T,
$$

where $r_{s}=s-\mu+1>0$.

\section{Difference Between Approximate and Actual Solutions}

In this section, we will estimate the difference between the approximate and actual solutions.

Let $\left(u_{\omega, \lambda}(t, x), \rho_{\omega, \lambda}(t, x)\right)$ be the solution to system (5) with initial data the value of the approximate solution $\left(u^{\omega, \lambda}(t, x), \rho^{\omega, \lambda}(t, x)\right)$ at time zero, that is, $\left(u_{\omega, \lambda}(t, x), \rho_{\omega, \lambda}(t, x)\right)$ satisfies

$$
\begin{cases}\partial_{t} u_{\omega, \lambda}-u_{\omega, \lambda} \partial_{x} u_{\omega, \lambda}-\partial_{x} \Lambda^{-2}\left(\frac{k_{1}}{2} u_{\omega, \lambda}^{2}+\frac{3-k_{1}}{2}\left(\partial_{x} u_{\omega, \lambda}\right)^{2}+\frac{k_{2}}{2} \rho_{\omega, \lambda}^{2}\right)=0, & t>0, x \in \mathbb{S}, \\ \partial_{t} \rho_{\omega, \lambda}-k_{3} u_{\omega, \lambda} \lambda \rho_{\omega, \lambda}-k_{3} \partial_{x} u_{\omega, \lambda} \rho_{\omega, \lambda}=0, & t>0, x \in \mathbb{S}, \\ u_{\omega, \lambda}(0, x)=u^{\omega, \lambda}(0, x)=\omega \lambda^{-1}+\lambda^{-s} \cos (\lambda x), & x \in \mathbb{S}, \\ \rho_{\omega, \lambda}(0, x)=\rho^{\omega, \lambda}(0, x)=\omega \lambda^{-1}+\lambda^{-s+1} \cos (\lambda x), & x \in \mathbb{S} .\end{cases}
$$


Note that $\left(u_{\omega, \lambda}(0, x), \rho_{\omega, \lambda}(0, x)\right) \in H^{s} \times H^{s-1}, s \geq 0$. Moreover, we have

$$
\begin{aligned}
\left\|u_{\omega, \lambda}(0, x)\right\|_{H^{s}} & \lesssim|\omega| \lambda^{-1}+1, \quad \lambda \gg 1, \\
\left\|\rho_{\omega, \lambda}(0, x)\right\|_{H^{s-1}} & \lesssim|\omega| \lambda^{-1}+1, \quad \lambda \gg 1 .
\end{aligned}
$$

Therefore, if $s>\frac{5}{2}$, by using Theorems 2.1 and 2.2, we have that for any $\omega$ in a bounded set and $\lambda \gg 1$, problem (11) has a unique solution $z_{\omega, \lambda} \in C\left([0, T] ; H^{s}\right) \times$ $C\left([0, T] ; H^{s-1}\right)$ with

$$
T \gtrsim \frac{1}{\left\|z_{\omega, \lambda}(0)\right\|_{H^{s} \times H^{s-1}}} \gtrsim \frac{1}{1+\lambda^{-1}} \gtrsim 1 .
$$

To estimate the difference between the approximate and actual solutions, we let

$$
v=u^{\omega, \lambda}-u_{\omega, \lambda}, \quad \sigma=\rho^{\omega, \lambda}-\rho_{\omega, \lambda} .
$$

Then $(v, \sigma)$ satisfies

$$
\begin{cases}v_{t}-\frac{1}{2} \partial_{x}\left[\left(u^{\omega, \lambda}+u_{\omega, \lambda}\right) v\right]-\partial_{x} \Lambda^{-2}\left[\frac{k_{1}}{2}\left(u^{\omega, \lambda}+u_{\omega, \lambda}\right) v\right. & \\ \left.\quad+\frac{3-k_{1}}{2}\left(u_{x}^{\omega, \lambda}+\partial_{x} u_{\omega, \lambda}\right) v_{x}+\frac{k_{2}}{2} \sigma\left(\rho^{\omega, \lambda}+\rho^{\omega, \lambda}\right)\right]=E, & t>0, x \in \mathbb{S}, \\ \sigma_{t}-k_{3} \rho_{x}^{\omega, \lambda} v-k_{3} \rho^{\omega, \lambda} v_{x}-k_{3} \sigma \partial_{x} u_{\omega, \lambda}-k_{3} \sigma_{x} u_{\omega, \lambda}=F, & t>0, x \in \mathbb{S}, \\ v(0, x)=\sigma(0, x)=0, & x \in \mathbb{S},\end{cases}
$$

where $E$ and $F$ are defined as in Sect. 3. Now we prove that the $H^{\mu} \times H^{\mu-1}$-norm of difference decays.

Theorem 4.1 Let $1<\mu<\frac{3}{2}$ and $s>\frac{5}{2}>\mu+1$, then

$$
\|v(t)\|_{H^{\mu}},\|\sigma(t)\|_{H^{\mu-1}} \lesssim \lambda^{-r_{s}}, \quad 0 \leq t \leq T, \lambda \gg 1,
$$

where $r_{s}=s-\mu+1-\frac{1}{2} \delta>0$.

Proof Note that

$$
\begin{gathered}
\frac{1}{2} \frac{d}{d t}\|v(t)\|_{H^{\mu}}^{2}=\int_{\mathbb{S}} \Lambda^{\mu} v_{t} \cdot \Lambda^{\mu} v \mathrm{~d} x, \\
\frac{1}{2} \frac{d}{d t}\|\sigma(t)\|_{H^{\mu-1}}^{2}=\int_{\mathbb{S}} \Lambda^{\mu-1} \sigma_{t} \cdot \Lambda^{\mu-1} \sigma \mathrm{d} x .
\end{gathered}
$$

Applying the operators $\Lambda^{\mu}$ and $\Lambda^{\mu-1}$ to both sides of the first and second equations of system (13), respectively, and integrating them, we have 


$$
\begin{aligned}
& \frac{1}{2} \frac{d}{d t}\|v(t)\|_{H^{\mu}}^{2}= \int_{\mathbb{S}} \Lambda^{\mu} E \cdot \Lambda^{\mu} v \mathrm{~d} x+\frac{1}{2} \int_{\mathbb{S}} \partial_{x} \Lambda^{\mu}\left[\left(u^{\omega, \lambda}+u_{\omega, \lambda}\right) v\right] \Lambda^{\mu} v \mathrm{~d} x \\
&+\int_{\mathbb{S}} \partial_{x} \Lambda^{\mu-2}\left[\frac{k_{1}}{2}\left(u^{\omega, \lambda}+u_{\omega, \lambda}\right) v\right. \\
&\left.+\frac{3-k_{1}}{2}\left(u_{x}^{\omega, \lambda}+\partial_{x} u_{\omega, \lambda}\right) v_{x}+\frac{k_{2}}{2} \sigma\left(\rho^{\omega, \lambda}+\rho_{\omega, \lambda}\right)\right] \Lambda^{\mu} v \mathrm{~d} x \\
& \frac{1}{2} \frac{d}{d t}\|\sigma(t)\|_{H^{\mu-1}}^{2}=\int_{\mathbb{S}} \Lambda^{\mu-1} F \cdot \Lambda^{\mu-1} \sigma \mathrm{d} x+k_{3} \int_{\mathbb{S}} \Lambda^{\mu-1} \partial_{x}\left(u_{\omega, \lambda} \sigma+v \rho^{\omega, \lambda}\right) \Lambda^{\mu-1} \sigma \mathrm{d} x
\end{aligned}
$$

Applying the Cauchy-Schwarz inequality in the first integral of right side of (16) and (17), we get

$$
\begin{aligned}
& \left|\int_{\mathbb{S}} \Lambda^{\mu} E \cdot \Lambda^{\mu} v \mathrm{~d} x\right| \leq\|E\|_{H^{\mu}}\|v(t)\|_{H^{\mu}}, \\
& \left|\int_{\mathbb{S}} \Lambda^{\mu-1} F \cdot \Lambda^{\mu-1} \sigma \mathrm{d} x\right| \leq\|F\|_{H^{\mu-1}}\|\sigma(t)\|_{H^{\mu-1}} .
\end{aligned}
$$

In order to estimate the other terms in (16) and (17), we need the following lemma.

Lemma 4.1 [20, Lemma 3] If $\sigma+1 \geq 0$, then

$$
\left\|\left[\Lambda^{\sigma} \partial_{x}, f\right] g\right\|_{L^{2}} \leq C\|f\|_{H^{\rho}}\|g\|_{H^{\sigma}}
$$

provided that $\rho>3 / 2$ and $\sigma+1 \leq \rho$.

In [20], the authors obtained that

$$
\begin{aligned}
& \left|\int_{\mathbb{S}} \partial_{x} \Lambda^{\mu}\left[\left(u^{\omega, \lambda}+u_{\omega, \lambda}\right) v\right] \Lambda^{\mu} v \mathrm{~d} x\right| \\
& \quad \lesssim\left(\left\|u^{\omega, \lambda}\right\|_{H^{\gamma}}+\left\|u_{\omega, \lambda}\right\|_{H^{\gamma}}\right)\|v(t)\|_{H^{\mu}},
\end{aligned}
$$

where $3 / 2<\gamma<s$. We remark that, in paper [20], the authors considered the periodic Camassa-Holm equation, but it is no difference between the periodic and line. Next, we estimate the third term of right side of (16). Firstly, by Lemma 2.2, we have

$$
\begin{aligned}
& \left|\int_{\mathbb{S}} \partial_{x} \Lambda^{\mu-2}\left[\frac{k_{1}}{2}\left(u^{\omega, \lambda}+u_{\omega, \lambda}\right) v\right] \Lambda^{\mu} v \mathrm{~d} x\right| \\
& \quad \lesssim\left\|\left(u^{\omega, \lambda}+u_{\omega, \lambda}\right) v\right\|_{H^{\mu-1}}\|v\|_{H^{\mu}} \\
& \quad \lesssim\left(\left\|u^{\omega, \lambda}+u_{\omega, \lambda}\right\|_{L^{\infty}}\|v\|_{H^{\mu-1}}+\|v\|_{L^{\infty}}\left\|u^{\omega, \lambda}+u_{\omega, \lambda}\right\|_{H^{\mu-1}}\right)\|v\|_{H^{\mu}} \\
& \quad \lesssim\left(\left\|u_{\omega, \lambda}\right\|_{H^{\mu}}+\left\|u^{\omega, \lambda}\right\|_{H^{\mu}}\right)\|v\|_{H^{\mu}}^{2} .
\end{aligned}
$$

By Lemma 4.1, we have 


$$
\begin{aligned}
\mid \int_{\mathbb{S}} & {\left[\frac{3-k_{1}}{2}\left(u_{x}^{\omega, \lambda}+\partial_{x} u_{\omega, \lambda}\right) v_{x}+\frac{k_{2}}{2} \sigma\left(\rho^{\omega, \lambda}+\rho_{\omega, \lambda}\right)\right] \Lambda^{\mu} v \mathrm{~d} x \mid } \\
\lesssim & \left|\int_{\mathbb{S}}\left[\Lambda^{\mu-2} \partial_{x},\left(u_{x}^{\omega, \lambda}+\partial_{x} u_{\omega, \lambda}\right)\right] v_{x} \cdot \Lambda^{\mu} v \mathrm{~d} x+\int_{\mathbb{S}}\left(u_{x}^{\omega, \lambda}+\partial_{x} u_{\omega, \lambda}\right) \Lambda^{\mu-2} \partial_{x} v_{x} \cdot \Lambda^{\mu} v \mathrm{~d} x\right| \\
& +\left|\int_{\mathbb{S}}\left[\Lambda^{\mu-2} \partial_{x},\left(\rho^{\omega, \lambda}+\rho_{\omega, \lambda}\right)\right] \sigma \cdot \Lambda^{\mu} v \mathrm{~d} x+\int_{\mathbb{S}}\left(\rho^{\omega, \lambda}+\rho_{\omega, \lambda}\right) \Lambda^{\mu-2} \partial_{x} \sigma \cdot \Lambda^{\mu} v \mathrm{~d} x\right| \\
\lesssim & \left.\left\|u^{\omega, \lambda}\right\|_{H^{\gamma+1}}+\left\|u_{\omega, \lambda}\right\|_{H^{\gamma+1}}\right)\|v\|_{H^{\mu}}^{2}+\left(\left\|\rho^{\omega, \lambda}\right\|_{H^{\gamma}}+\left\|\rho_{\omega, \lambda}\right\|_{H^{\gamma}}\right)\left(\|v\|_{H^{\mu}}^{2}+\|\sigma\|_{H^{\mu-1}}^{2}\right),
\end{aligned}
$$

where $\gamma$ satisfies $3 / 2<\gamma<s-1$. We remark there exists $\gamma$ satisfying $3 / 2<\gamma<s-1$ because the index $s$ considered here is strictly lager than $5 / 2$.

Integrating by part and using Lemma 4.1, we have

$$
\begin{aligned}
& \left|k_{3} \int_{\mathbb{S}} \Lambda^{\mu-1} \partial_{x}\left(u_{\omega, \lambda} \sigma+v \rho^{\omega, \lambda}\right) \Lambda^{\mu-1} \sigma \mathrm{d} x\right| \\
& \lesssim\left\|v \rho^{\omega, \lambda}\right\|_{H^{\mu}}\|\sigma\|_{H^{\mu-1}}+\mid \int_{\mathbb{S}}\left[\Lambda^{\mu-1} \partial_{x}, u_{\omega, \lambda}\right] \sigma \cdot \Lambda^{\mu-1} \sigma \mathrm{d} x \\
& \quad+\int_{\mathbb{S}} u_{\omega, \lambda} \partial_{x} \Lambda^{\mu-1} \sigma \cdot \Lambda^{\mu-1} \sigma \mathrm{d} x \mid \\
& \lesssim\left\|\rho^{\omega, \lambda}\right\|_{H^{\mu}}\|v\|_{H^{\mu}}\|\sigma\|_{H^{\mu-1}} \\
& \quad+\left\|\left[\Lambda^{\mu-1} \partial_{x}, u_{\omega, \lambda}\right] \sigma\right\|_{L^{2}}\|\sigma\|_{H^{\mu-1}}+\left|\int_{\mathbb{S}} \partial_{x} u_{\omega, \lambda}\left(\Lambda^{\mu-1} \sigma\right)^{2} \mathrm{~d} x\right| \\
& \quad \lesssim\left(\left\|\rho^{\omega, \lambda}\right\|_{H^{\mu}}+\left\|u_{\omega, \lambda}\right\|_{H^{\gamma}}\right)\left(\|v\|_{H^{\mu}}^{2}+\|\sigma\|_{H^{\mu-1}}^{2}\right) .
\end{aligned}
$$

Substituting (18)-(20) into (16) and (17), and adding the resulting equations, we get

$$
\begin{aligned}
& \frac{1}{2} \frac{d}{d t}\left(\|v(t)\|_{H^{\mu}}^{2}+\|\sigma(t)\|_{H^{\mu-1}}^{2}\right) \\
& \quad \lesssim\|E\|_{H^{\mu}}\|v(t)\|_{H^{\mu}}+\|F\|_{H^{\mu-1}}\|\sigma(t)\|_{H^{\mu-1}} \\
& \quad+\left(\left\|\rho^{\omega, \lambda}\right\|_{H^{\gamma}}+\left\|\rho_{\omega, \lambda}\right\|_{H^{\gamma}}+\left\|u^{\omega, \lambda}\right\|_{H^{\gamma+1}}+\left\|u_{\omega, \lambda}\right\|_{H^{\gamma+1}}\right)\left(\|v\|_{H^{\mu}}^{2}+\|\sigma\|_{H^{\mu-1}}^{2}\right) .
\end{aligned}
$$

Note that

$$
u^{\omega, \lambda}=\omega \lambda^{-1}+\lambda^{-s} \cos (\lambda x+\omega t), \quad \rho^{\omega, \lambda}=\omega \lambda^{-1}+\lambda^{-s+1} \cos \left(\lambda x+k_{3} \omega t\right),
$$

we have

$$
\begin{aligned}
\left\|u^{\omega, \lambda}\right\|_{H^{\gamma+1}} & \lesssim \lambda^{-1}+\lambda^{-s+\gamma+1}, \\
\left\|\rho^{\omega, \lambda}\right\|_{H^{\gamma}} & \lesssim \lambda^{-1}+\lambda^{-s+\gamma+1} .
\end{aligned}
$$

By (9), we have

$$
\left\|u_{\omega, \lambda}(t)\right\|_{H^{s}},\left\|\rho_{\omega, \lambda}(t)\right\|_{H^{s-1}} \leq\left\|z_{\omega, \lambda}(t)\right\|_{H^{s} \times H^{s-1}} \lesssim\left\|z_{\omega, \lambda}(0)\right\|_{H^{s} \times H^{s-1}},
$$

where $z_{\omega, \lambda}(t)=\left(u_{\omega, \lambda}(t), \rho_{\omega, \lambda}(t)\right)$. Since $z_{\omega, \lambda}(0)=z^{\omega, \lambda}(0)$, we get 


$$
\begin{aligned}
\left\|u_{\omega, \lambda}(t)\right\|_{H^{\gamma+1}},\left\|\rho_{\omega, \lambda}(t)\right\|_{H^{\gamma}} \\
\quad \lesssim\left\|z^{\omega, \lambda}(0)\right\|_{H^{\gamma+1} \times H^{\gamma}} \\
\quad \lesssim\left\|u^{\omega, \lambda}(0)\right\|_{H^{\gamma+1}}+\left\|\rho^{\omega, \lambda}(0)\right\|_{H^{\gamma}} \\
\quad \lesssim \lambda^{-1}+\lambda^{-s+\gamma+1} .
\end{aligned}
$$

Let $\tilde{z}(t, x)=(v(t, x), \sigma(t, x))$ and $\|\tilde{z}(t)\|_{H^{\mu} \times H^{\mu-1}}^{2}=\|v(t)\|_{H^{\mu}}^{2}+\|\sigma(t)\|_{H^{\mu-1}}^{2}$, then by (21), we obtain that

$$
\begin{aligned}
\frac{1}{2} \frac{d}{d t}\|\tilde{z}(t)\|_{H^{\mu} \times H^{\mu-1}}^{2} & \lesssim\left(\|E\|_{H^{\mu}}+\|F\|_{H^{\mu-1}}\right)\|\tilde{z}(t)\|_{H^{\mu} \times H^{\mu-1}}+\lambda^{-\rho_{s}}\|\tilde{z}(t)\|_{H^{\mu} \times H^{\mu-1}}^{2} \\
& \lesssim \lambda^{-r_{s}}\|\tilde{z}(t)\|_{H^{\mu} \times H^{\mu-1}}+\lambda^{-s+\gamma+1}\|\tilde{z}(t)\|_{H^{\mu} \times H^{\mu-1}}^{2}, \quad \lambda \gg 1,
\end{aligned}
$$

where we have used Theorem 3.1. Consequently,

$$
\frac{d}{d t}\|\tilde{z}(t)\|_{H^{\mu} \times H^{\mu-1}} \lesssim \lambda^{-s+\gamma+1}\|\tilde{z}(t)\|_{H^{\mu} \times H^{\mu-1}}+\lambda^{-r_{s}}, \quad \lambda \gg 1 .
$$

Since $\|\tilde{z}(0)\|_{H^{\mu} \times H^{\mu-1}}=\left(\|v(0)\|_{H^{\mu}}^{2}+\|\sigma(0)\|_{H^{\mu-1}}^{2}\right)^{1 / 2}=0$ and for $s>5 / 2$, we can choose $\gamma$ such that $-s+\gamma+1<0$, by (22) and Gronwall's inequality, we obtain

$$
\|\tilde{z}(t)\|_{H^{\mu} \times H^{\mu-1}} \lesssim \lambda^{-r_{s}}, \quad 0 \leq t \leq T, \lambda \gg 1 .
$$

Note that

$$
\|v(t)\|_{H^{\mu}}, \quad\|\sigma(t)\|_{H^{\mu-1}} \leq\|\tilde{z}(t)\|_{H^{\mu} \times H^{\mu-1}},
$$

we see that

$$
\|v(t)\|_{H^{\mu}}, \quad\|\sigma(t)\|_{H^{\mu-1}} \lesssim \lambda^{-r_{s}}, \quad 0 \leq t \leq T, \lambda \gg 1 .
$$

This completes the proof of this theorem.

\section{Non-uniform Dependence}

In this section, we will prove non-uniform dependence for system (5) by taking advantage of the information provided by Theorems 2.1, 2.2, 3.1 and 4.1. Our main result is the following theorem.

Theorem 5.1 If $s>5 / 2$, then the data-to-solution $z(0) \rightarrow z(t)$ for system (5) is not uniformly continuous from any bounded subset of $H^{s} \times H^{s}$ into $C\left([-T, T] ; H^{s}\right) \times C\left([-T, T] ; H^{s-1}\right)$, where $z(0)=\left(u_{0}(x), \rho_{0}(x)\right)$ and $z(t)=(u(t, x), \rho(t, x))$. More precisely, there exist two sequences of solutions $\left(u_{\lambda}(t), \rho_{\lambda}(t)\right)$ and $\left(\tilde{u}_{\lambda}(t), \tilde{\rho}_{\lambda}(t)\right)$ to the differential equations of $(5)$ in $C\left([-T, T] ; H^{s}\right) \times C\left([-T, T] ; H^{s-1}\right)$ such that 


$$
\begin{aligned}
& \left\|u_{\lambda}(t)\right\|_{H^{s}}+\left\|\tilde{u}_{\lambda}(t)\right\|_{H^{s}}+\left\|\rho_{\lambda}(t)\right\|_{H^{s-1}}+\left\|\tilde{\rho}_{\lambda}(t)\right\|_{H^{s-1}} \lesssim 1, \\
& \quad \lim _{\lambda \rightarrow \infty}\left\|u_{\lambda}(0)-\tilde{u}_{\lambda}(0)\right\|_{H^{s}}=\lim _{\lambda \rightarrow \infty}\left\|\rho_{\lambda}(0)-\tilde{\rho}_{\lambda}(0)\right\|_{H^{s-1}}=0,
\end{aligned}
$$

and

$$
\liminf _{\lambda \rightarrow \infty}\left(\left\|u_{\lambda}(t)-\tilde{u}_{\lambda}(t)\right\|_{H^{s}}+\left\|\rho_{\lambda}(t)-\tilde{\rho}_{\lambda}(t)\right\|_{H^{s-1}}\right) \gtrsim \sin t, \quad|t|<T \leq 1 .
$$

Proof Let $\left(u_{\lambda}(t), \rho_{\lambda}(t)\right)=\left(u_{1, \lambda}(t, x), \rho_{1, \lambda}(t, x)\right) \operatorname{and}\left(\tilde{u}_{\lambda}(t), \tilde{\rho}_{\lambda}(t)\right)=\left(u_{-1, \lambda}(t, x), \rho_{-1, \lambda}(t, x)\right)$, where $\left(u_{1, \lambda}(t, x), \rho_{1, \lambda}(t, x)\right)$ and $\left(u_{-1, \lambda}(t, x), \rho_{-1, \lambda}(t, x)\right)$ are the unique solution to Cauchy problem (11) with initial data $\left(u^{1, \lambda}(0, x), \rho^{1, \lambda}(0, x)\right)$ and $\left(u^{-1, \lambda}(0, x), \rho^{-1, \lambda}(0, x)\right)$, respectively.

It follows from Theorem 2.1 that these solutions belong in $C\left([0, T] ; H^{s}\right) \times C\left([0, T] ; H^{s-1}\right)$. By (12) and the assumptions after Theorem 2.1, we see that $T$ is independent of $\lambda \gg 1$. Letting $k=[s]+2$ and using estimate (9), we have

$$
\left\|u_{ \pm 1, \lambda}(t)\right\|_{H^{k}},\left\|\rho_{ \pm 1, \lambda}(t)\right\|_{H^{k}} \lesssim\left\|z^{ \pm 1, \lambda}(0)\right\|_{H^{k} \times H^{k-1}},
$$

where

$$
z^{ \pm 1, \lambda}(0)=\left(u^{ \pm 1, \lambda}(0), \rho^{ \pm 1, \lambda}(0)\right)
$$

$\left\|z^{ \pm 1, \lambda}(0)\right\|_{H^{k} \times H^{k-1}}^{2}=\left\|u^{ \pm 1, \lambda}(0)\right\|_{H^{k}}^{2}+\left\|\rho^{ \pm 1, \lambda}(0)\right\|_{H^{k-1}}^{2}$. If $\lambda$ is large enough, then from Lemma 3.1 we have

$$
\begin{aligned}
\left\|u^{ \pm 1, \lambda}(t)\right\|_{H^{k}} & \leq\left\|\omega \lambda^{-1}\right\|_{H^{k}}+\lambda^{-s}\|\cos (\lambda x+\omega t)\|_{H^{k}} \\
& \lesssim \lambda^{-1}+\lambda^{k-s} \\
\left\|\rho^{ \pm 1, \lambda}(t)\right\|_{H^{k-1}} & \lesssim \lambda^{-1}+\lambda^{k-s}
\end{aligned}
$$

which gives

$$
\left\|u^{ \pm 1, \lambda}(t)\right\|_{H^{k}},\left\|\rho^{ \pm 1, \lambda}(t)\right\|_{H^{k-1}} \lesssim \lambda^{k-s} .
$$

Combining (25) with (26), we obtain

$$
\left\|u_{ \pm 1, \lambda}(t)\right\|_{H^{k}},\left\|\rho_{ \pm 1, \lambda}(t)\right\|_{H^{k-1}} \lesssim \lambda^{k-s}, \quad \lambda \gg 1 .
$$

Estimate (26) together with (27) yield

$$
\left\|u^{ \pm 1, \lambda}(t)-u_{ \pm 1, \lambda}(t)\right\|_{H^{k}},\left\|\rho^{ \pm 1, \lambda}(t)-\rho_{ \pm 1, \lambda}(t)\right\|_{H^{k-1}} \lesssim \lambda^{k-s}, \quad \lambda \gg 1 .
$$

Theorem 4.1 implies that

$$
\left\|u^{ \pm 1, \lambda}(t)-u_{ \pm 1, \lambda}(t)\right\|_{H^{\mu}},\left\|\rho^{ \pm 1, \lambda}(t)-\rho_{ \pm 1, \lambda}(t)\right\|_{H^{\mu-1}} \lesssim \lambda^{-r_{s}}, \quad \lambda \gg 1 .
$$

Now, applying the interpolation inequality

$$
\|\varphi\|_{H^{s}} \leq\|\varphi\|_{H^{s_{1}}}^{\left(s_{2}-s\right) /\left(s_{2}-s_{1}\right)}\|\varphi\|_{H^{s_{2}}}^{\left(s-s_{1}\right) /\left(s_{2}-s_{1}\right)}
$$

with $s_{1}=\mu$ and $s_{2}=[s]+2=k$, and using estimates (28) and (29), we get 


$$
\begin{aligned}
\left\|u^{ \pm 1, \lambda}(t)-u_{ \pm 1, \lambda}(t)\right\|_{H^{s}} & \leq\left\|u^{ \pm 1, \lambda}(t)-u_{ \pm 1, \lambda}(t)\right\|_{H^{\mu}}^{(k-s) /(k-\mu)}\left\|u^{ \pm 1, \lambda}(t)-u_{ \pm 1, \lambda}(t)\right\|_{H^{k}}^{(s-\mu) /(k-\mu)} \\
& \lesssim \lambda^{-r_{s}(k-s) /(k-\mu)} \lambda^{(k-s)(s-\mu) /(k-\mu)} \\
& \lesssim \lambda^{-\left(r_{s}-s+\mu\right)(k-s) /(k-\mu)}, \quad \lambda \gg 1 .
\end{aligned}
$$

Hence

$$
\left\|u^{ \pm 1, \lambda}(t)-u_{ \pm 1, \lambda}(t)\right\|_{H^{s}} \lesssim \lambda^{-\varepsilon_{s}}, \quad \lambda \gg 1
$$

where $\varepsilon_{s}=1 /(s+2)$.

Now, we prove (23) and (24). We only consider $u_{ \pm 1, \lambda}(t, x)$ because $\rho_{ \pm 1, \lambda}(t, x)$ can be similarly proved. Firstly, we have

$$
\left\|u_{1, \lambda}(0)-u_{-1, \lambda}(0)\right\|_{H^{s}}=2 \lambda^{-1}\|\omega\|_{H^{s}} \rightarrow 0 \quad \text { as } \lambda \rightarrow \infty,
$$

which implies that (23) holds. Next, we prove (24). It is easy to see that

$$
\liminf _{\lambda \rightarrow \infty}\left(\left\|u_{\lambda}(t)-\tilde{u}_{\lambda}(t)\right\|_{H^{s}}+\left\|\rho_{\lambda}(t)-\tilde{\rho}_{\lambda}(t)\right\|_{H^{s-1}}\right) \geq \liminf _{\lambda \rightarrow \infty}\left\|u_{\lambda}(t)-\tilde{u}_{\lambda}(t)\right\|_{H^{s}} .
$$

Thus we only prove that

$$
\liminf _{\lambda \rightarrow \infty}\left\|u_{\lambda}(t)-\tilde{u}_{\lambda}(t)\right\|_{H^{s}} \gtrsim \sin t, \quad|t|<T \leq 1 .
$$

Obviously,

$$
\left\|u_{1, \lambda}(t)-u_{-1, \lambda}(t)\right\|_{H^{s}} \geq\left\|u^{1, \lambda}(t)-u^{-1, \lambda}(t)\right\|_{H^{s}}-\left\|u^{1, \lambda}(t)-u_{1, \lambda}(t)\right\|_{H^{s}}-\left\|u^{-1, \lambda}(t)-u_{-1, \lambda}(t)\right\|_{H^{s}} .
$$

It follows from (30) that

$$
\left\|u_{1, \lambda}(t)-u_{-1, \lambda}(t)\right\|_{H^{s}} \geq\left\|u^{1, \lambda}(t)-u^{-1, \lambda}(t)\right\|_{H^{s}}-c \lambda^{-\varepsilon_{s}}, \quad \lambda \gg 1,
$$

which implies that

$$
\liminf _{\lambda \rightarrow \infty}\left\|u_{1, \lambda}(t)-u_{-1, \lambda}(t)\right\|_{H^{s}} \geq \liminf _{\lambda \rightarrow \infty}\left\|u^{1, \lambda}(t)-u^{-1, \lambda}(t)\right\|_{H^{s}} .
$$

Using the identity

$$
\cos \alpha-\cos \beta=-2 \sin \frac{\alpha+\beta}{2} \sin \frac{\alpha-\beta}{2}
$$

gives

$$
u^{1, \lambda}(t)-u^{-1, \lambda}(t)=2 \lambda^{-1}-2 \lambda^{-s} \sin (\lambda x) \sin t
$$

Thus,

$$
\begin{aligned}
\left\|u^{1, \lambda}(t)-u^{-1, \lambda}(t)\right\|_{H^{s}} & \geq 2 \lambda^{-s}\|\sin \lambda x\|_{H^{s}}|\sin t|-2 \lambda^{-1}\|1\|_{H^{s}} \\
& \gtrsim \lambda^{-s}\|\sin \lambda x\|_{H^{s}}|\sin t|-\lambda^{-1}, \quad \lambda \gg 1 .
\end{aligned}
$$


Letting $\lambda \rightarrow \infty$ in the above inequality, we have

$$
\liminf _{\lambda \rightarrow \infty}\left\|u^{1, \lambda}(t)-u^{-1, \lambda}(t)\right\|_{H^{s}} \gtrsim|\sin t| .
$$

Summing inequalities (31) and (32) up, it yields inequality (24). This completes the proof of this theorem.

\section{Conclusion}

In this paper, we considered the initial date problem of a periodic two-component b-family system. The solution map of the Cauchy problem of the b-family system is not uniformly continuous in $H^{s}(\mathbb{S}), s>5 / 2$, is obtained.

Comparing with the earlier results, we studied a more general shallow water equation. By constructing the approximating solutions, we prove the solution map is continuous but not uniformly continuous in $H^{s}$.

Open Access This article is distributed under the terms of the Creative Commons Attribution 4.0 International License (http://creativecommons.org/licenses/by/4.0/), which permits unrestricted use, distribution, and reproduction in any medium, provided you give appropriate credit to the original author(s) and the source, provide a link to the Creative Commons license, and indicate if changes were made.

Author Contributions All authors contributed to the writing of the present article and they read and approved the final manuscript.

Funding This work was supported by NSFC of China grants 11901158, and the Startup Foundation for Introducing Talent of NUIST.

Availability of Data and Material Not applicable.

\section{Declarations}

Conflict of Interest The authors declare that they have no competing interests.

Ethics Approval Not applicable.

Consent to Participate Not applicable.

Consent for Publication Not applicable.

Open Access This article is licensed under a Creative Commons Attribution 4.0 International License, which permits use, sharing, adaptation, distribution and reproduction in any medium or format, as long as you give appropriate credit to the original author(s) and the source, provide a link to the Creative Commons licence, and indicate if changes were made. The images or other third party material in this article are included in the article's Creative Commons licence, unless indicated otherwise in a credit line to the material. If material is not included in the article's Creative Commons licence and your intended use is not permitted by statutory regulation or exceeds the permitted use, you will need to obtain permission directly from the copyright holder. To view a copy of this licence, visit http://creativecommons.org/licen ses/by/4.0/. 


\section{References}

1. Guha, P.: Euler-Poincare formalism of (two component) Degasperis-Procesi and Holm-Staley type system. J. Nonlinear Math. Phys. 14, 398-429 (2007)

2. Liu, J., Yin, Z.: On the Cauchy problem $b$-family system. Nonlinear Anal. RWA 12, 3608-3620 (2011)

3. Zou, X.: Properties of the solutions to the two-component $b$-family systems. Nonlinear Anal. 75, 6250-6259 (2012)

4. Zhu, M., Xu, J.: On the Cauchy problem for the two-component b-family system. Math. Methods Appl. Sci. 36, 2154-2173 (2013)

5. Dullin, H., Gottwald, G., Holm, D.: Camassa-Holm, Korteweg-de Vries-5 and other asymptotically equivalent equations for shallow water waves. Fluid Dyn. Res. 33, 73-79 (2003)

6. Dullin, H., Gottwald, G., Holm, D.: On asymptotically equivalent shallow water wave equations. Physica D 190, 1-14 (2004)

7. Camassa, R., Holm, D.: An integrable shallow water equation with peaked solitons. Phys. Rev. Lett. 71, 1661-1664 (1993)

8. Constantin, A., Escher, J.: Well-posedness, global existence and blowup phenomena for a periodic quasi-linear hyperbolic equation. Commun. Pure Appl. Math. 51, 475-504 (1998)

9. Bressan, A., Constantin, A.: Global conservation solutions of the Camassa-Holm equation. Arch. Ration. Mech. Anal. 183, 215-239 (2007)

10. Escher, J., Yin, Z.: Initial boundary value problems of the Camassa-Holm equation. Commun. Partial Differ. Equ. 33, 377-395 (2008)

11. Tian, L.X., Shen, C.Y., Ding, D.P.: Optimal control of the viscous Camassa-Holm equation. Nonlinear Anal. Real World Appl. 10, 519-530 (2009)

12. Degasperis, A., Procesi, M.: Asymptotic integrability. In: Symmetry and Perturbation Theory, Rome, 1998, pp. 23-37. World Scientific Publishing, River Edge (1999)

13. Yin, Z.: Global existence for a new periodic integrable equation. J. Math. Anal. Appl. 283, 129-139 (2003)

14. Holm, D.D., Naraigh, L., Tronci, C.: Singular solution of a modified two component CamassaHolm equation. Phy. Rev. E 79, 1-13 (2009)

15. Constantin, A., Ivanov, R.: On an integrable two-component Camassa-Holm shallow water system. Phys. Lett. A 372, 7129-7132 (2008)

16. Escher, J., Lechtenfeld, O., Yin, Z.: Well-posedness and blow-up phenomena for the 2-component Camassa-Holm equation. Discret. Contin. Dyn. Syst. 19, 493-513 (2007)

17. Himonas, A., Misiolek, G., Ponce, G., Zhou, Y.: Persistence properties and unique continuous of solutions of the Camassa-Holm equation. Commun. Math. Phys. 271, 511-522 (2007)

18. Wu, S., Yin, Z.: Blow-up and decay of the solution of the weakly dissipative Degasperis-Procesi equation. SIAM J. Math. Anal. 40, 475-490 (2008)

19. Himonas, A., Kenig, C.: Non-uniform dependence on initial data for the $\mathrm{CH}$ equation on the line. Differ. Integral Equ. 22, 201-224 (2009)

20. Himonas, A., Kenig, C., Misiolek, G.: Non-uniform dependence for the periodic CH equation. Commun. Partial Differ. Equ. 35, 1145-1162 (2010)

21. Lv, G.Y., Pang, P.Y.H., Wang, M.X.: Non-uniform dependence on initial data for the $\mu$ - $b$ equation. Z. Angew. Math. Phys. 64, 1543-1554 (2013)

22. Lv, G.Y., Wang, M.X.: Non-uniform dependence for a modified Camassa-Holm system. J. Math. Phys. 53, 013101 (2012)

23. Lv, G.Y., Wang, X.H.: On Cauchy problem for a two-component b-family system. Nonlinear Anal. 11, 1-14 (2014)

24. Wang, X.H.: On initial data problem for the two-component Camassa-Holm system. Electron. J. Differ. Equ. 2014(148), 1-15 (2014)

25. Kato, T.: Quasi-linear equation of evolution, with application to partial differential equation. In: Spectral Theory and Differential Equations. Lecture Notes in Mathematics, vol. 448, pp. 25-70. Springer, Berlin (1975)

26. Iorio, R., de Magãlhaes Iorio: Fourier Analysis and Partial Differential Equation. Cambridge University Press, Cambridge (2001) 